\title{
TRAF4 promotes tumorigenesis of breast cancer through activation of Akt
}

\author{
JIE ZHANG ${ }^{1}$, XIAORUI LI ${ }^{2}$, WEILONG YANG ${ }^{3}$, XIAOKUI JIANG ${ }^{4}$ and NA LI \\ ${ }^{1}$ Department of Pathology, Xinxiang Medical University, Xinxiang, Henan 453003; ${ }^{2}$ Department of Oncology, \\ The First Affiliated Hospital of Xinxiang Medical University Xinxiang, Henan 453100; Departments of ${ }^{3}$ Neurosurgery \\ and ${ }^{4}$ General Surgery, The Third Affiliated Hospital of Xinxiang Medical University, Xinxiang, Henan 453003, P.R. China
}

Received April 22, 2014; Accepted June 16, 2014

DOI: $10.3892 /$ or.2014.3304

\begin{abstract}
Increasing evidence suggests that tumor necrosis factor receptor-associated factor 4 (TRAF4) is an oncogene which is frequently overexpressed in many human carcinomas. Although TRAF4 was originally identified in breast cancer, the underlying mechanism of TRAF4 in tumorigenesis remains largely unknown. In the present study, we found that TRAF4 was overexpressed in cancer cells, and RNA interference (RNAi)-mediated gene knockdown of TRAF4 decreased cell growth, cell migration and invasion. Next, we found that TRAF4 promoted cell survival kinase Akt membrane recruitment, which is essential for Akt activation. Furthermore, we demonstrated a direct interaction between Akt and TRAF4. Additionally, overexpression of constitutively activated Akt reversed cell growth arrest in TRAF4 gene-silenced cells. Taken together, our data indicate that TRAF4 plays an important role in tumorigenesis of breast cancer through direct interaction and activation of Akt, implying that TRAF4 may be a potential molecular target for breast cancer prevention and therapy.
\end{abstract}

\section{Introduction}

The tumor necrosis factor receptor-associated factor (TRAF) family, consisting of seven members (TRAF1-7), is a group of signaling adaptors which participate in various signaling pathways through binding of the tumor necrosis factor receptor superfamily (1-3). Two decades since the TRAF family was first cloned, marked progress in the understanding of the functions of the TRAF family has been acquired. TRAF family

Correspondence to: Dr Na Li, Department of Pathology, Xinxiang Medical University, 601 Jinsui Road, Hongqi, Xinxiang, Henan 453003, P.R. China

E-mail: nalixxmu@163.com

Abbreviations: TRAF4, tumor necrosis factor receptor associated factor 4; RNAi, RNA interference; BrdU, bromodeoxyuridine; EGF, epidermal growth factor

Key words: breast cancer, TRAF4, Akt, cell proliferation members have now been suggested to be signal transducers, modulating immune receptors, cytokine receptors, C-type lectin receptors and adaptive immune receptors (3). Therefore, the TRAF family has been demonstrated to contribute to the pathology of a variety of human diseases including cancers, autoimmune diseases and neurodegenerative diseases (4-6). Considering the importance of the TRAF family, targeting these molecules may lead to the development of therapeutic intervention of TRAF-mediated human diseases.

Among the seven members, TRAF4 is a unique member of the TRAF family, and is one of the conserved proteins during evolution (7). TRAF4 is an important gene in the regulation of an organism's development and has been reported to be involved in embryogenesis $(8,9)$. In addition, research has demonstrated that TRAF4 is required in central nervous system myelin homeostasis (10). TRAF4 deficiency is embryonic lethal and causes severe developmental abnormalities in the respiratory system and axial skeleton in mouse models $(11,12)$. Moreover, dendritic cells from TRAF4-deficient mice showed reduced cell migratory ability in vivo and in vitro (13). Interestingly, TRAF4 is the first member of the TRAF family that has been found to be overexpressed in cancers, and is now considered an oncogene $(14,15)$. TRAF4 was originally identified in a breast cancer cDNA library by gene screening to play an important role in the initiation and progression of primary breast cancers $(16,17)$. Studies have reported that TRAF4 has multiple subcellular localizations including cytoplasmic, nuclear, and membrane and have been described in the literature $(16,18,19)$. Particularly, the subcellular localization of TRAF4 in cancers is mainly in the cytoplasm or nucleus (18). Moreover, breast cancer patients with TRAF4 nuclear localization have poor survival due to its ability to destabilize the p53 protein in the nucleus (20).

As a signal transducer, studies have revealed that TRAF4 is engaged in several signaling pathways. Several studies have shown that TRAF4 is recruited to different tumor necrosis factor receptors and Toll-like receptors (21-23). It has been reported that TRAF4 regulates the glucocorticoid-induced $\mathrm{NF}-\kappa \mathrm{B}$ signaling pathway, implying its role in modulating the suppressive functions of Treg cells (24). TRAF4 promotes MEKK4 oligomerization to mediate Jun N-terminal kinase activation in embryos (25). In addition, TRAF4 is a negative regulator of IL-17 signaling which is involved in regulating 
Th17-mediated disease (26). In spite of these findings, the underlying mechanism of TRAF4 in tumorigenesis remains largely unknown.

In the present study, we aimed to explore the biological functions of TRAF4 in breast cancer cells. By using different breast cancer cells, we found that TRAF4 was particularly overexpressed. Knockdown of TRAF4 significantly inhibited cell proliferation and decreased cell migration and the invasion of breast cancer cells. More importantly, we identified that TRAF4 was capable of promoting Akt activation. Furthermore, a direct interaction was observed between TRAF4 and Akt which was essential for Akt membrane recruitment. Additionally, overexpression of constitutively active Akt reversed the cell growth arrest in TRAF4 knockdown cells. Taken together, our data support the suggestion that TRAF4 plays an important role in cancer cells through the activation of Akt, and may be a potential candidate molecular target for breast cancer prevention and therapy.

\section{Materials and methods}

Cell lines and cell culture. The human breast cancer cell lines MCF-7, T47D and MDA-MB-468 were obtained from the American Type Culture Collection (ATCC; Manassas, VA, USA). All cells were maintained according to standard protocols. Briefly, cells were maintained in Dulbecco's modified Eagle's medium (DMEM) supplemented with $10 \%$ fetal bovine serum (FBS) to which $100 \mathrm{U} / \mathrm{ml}$ of penicillin $\mathrm{G}, 0.1 \mathrm{mg} / \mathrm{ml}$ streptomycin sulfate and $0.25 \mu \mathrm{g} / \mathrm{ml}$ amphotericin B were added. Human mammary epithelial cells (HMECs) and 293T cells were cultured in DMEM containing 10\% FBS and 1\% antibiotics. All cells were cultured at $37^{\circ} \mathrm{C}$ with $5 \% \mathrm{CO}_{2}$ in an incubator (Life Technologies, Baltimore, MD, USA).

Plasmids and small interference RNA (siRNA) transfection. Cells were seeded in a 6 -well culture plate $\left(2 \times 10^{5}\right.$ cells/well $)$ under standard conditions. When the cells reached $80 \%$ confluency, cell transfection was performed as per the standard protocols of the manufacturer. Briefly, plasmid DNA [pCDNA3.0-TRAF4, Myc-Akt1 or constitutively active (CA)-Akt1; Addgene] or siRNA (TRAF siRNA, sc-36713; Santa Cruz Biotechnology, Santa Cruz, CA, USA) was diluted in $500 \mu 1$ of DMEM with $5 \mu 1$ Lipofectamine (Invitrogen, Carlsbad, CA, USA), before being mixed and incubated at room temperature for $15 \mathrm{~min}$. The mixtures were then added to the cells to a final volume of $3 \mathrm{ml}$ medium and incubated for 36 to $48 \mathrm{~h}$, before proteins were extracted for further analysis.

Bromodeoxyuridine (BrdU) assay. The BrdU cell proliferation assay kit (Millipore, Billerica, MA, USA) was used for cell proliferation analysis as per the manufacturer's instructions. Briefly, cells in 96-well plates were transfected with plasmids or siRNA for $24 \mathrm{~h}$, then BrdU solution (10 $\mu \mathrm{l} /$ well) was added and incubated for $2 \mathrm{~h}$. The old medium was discarded, the Fixing/Denaturing solution (100 $\mu 1 /$ well) was added and incubated at room temperature for $15 \mathrm{~min}$. After that, the supernatants were removed, and the prepared detection antibody solution (100 $\mu \mathrm{l} /$ well) was added for incubation at room temperature for $1 \mathrm{~h}$. Subsequently, plates were washed three times with wash buffer, and the prepared horseradish peroxidase (HRP)-conjugated secondary antibody solution (100 $\mu \mathrm{l} /$ well) was added and incubated for $30 \mathrm{~min}$ at room temperature. Then, plates were washed, and tetramethylbenzidine (TMB) substrate $(100 \mu \mathrm{l})$ was added for incubation at room temperature for $30 \mathrm{~min}$. The amount of BrdU incorporation into the cells was measured at $450 \mathrm{~nm}$ by a microplate reader (Bio-Rad, Hercules, CA, USA). Experiments were performed in quintuplicate and repeated three times.

Cell invasion and migration assays. Cells $\left(2 \times 10^{5}\right)$ were suspended in a volume of $50 \mu 1$ serum-free medium which wrer then plated in the upper chamber of chemotaxis chambers (Neuro Probe, Gaithersburg, MD, USA). Complete medium (75 $\mu \mathrm{l})$ was added to the lower chamber and incubated at $37^{\circ} \mathrm{C}$ for $48 \mathrm{~h}$; then, the inserts were removed and submerged in PBS to remove the unattached cells, before being fixed and stained by Diff Quick (IHC World, Bethesda, MD, USA). After that, membranes were cut and mounted on slides. Images were captured of the migrated cells (x20) on the underside of the membrane, and 10 visual fields in each membrane were randomly selected for cell number counting. For the invasion assays, cells $\left(5 \times 10^{5}\right)$ in $60 \mu \mathrm{l}$ serum-free medium were plated in the top compartment of Matrigel-coated invasion chambers $(8-\mu \mathrm{m}$ pore membrane). Fibroblast conditioned medium $(0.75 \mathrm{ml})$ was added to the bottom chambers, and cultures were incubated at $37^{\circ} \mathrm{C}$ for $48 \mathrm{~h}$. The membranes were fixed and stained using Diff Quick. Images were captured of the invaded cells (x20), and 10 visual fields in each membrane were randomly selected for cell number counting. Each assay was repeated 3 times independently.

Membrane fractionation. Cells were starved for $24 \mathrm{~h}$ in DMEM containing 0.1\% FBS in 6-well plates. Epidermal growth factor (EGF) $(50 \mathrm{ng} / \mathrm{ml})$ was added and incubated for $30 \mathrm{~min}$. After that, the membrane and cytosolic fractions were extracted using the Membrane Protein Extraction Kit (Sangon, Shanghai, China) according to the manufacturer's instructions. Briefly, cells were washed with wash buffer at least three times. Then, $1 \mathrm{ml}$ of extract buffer containing DTT $(1 \mu \mathrm{g} /$ $\mathrm{ml}$ ) was added and homogenized under an ice-cold condition followed by centrifugation $(14,000 \mathrm{rpm})$ at $4^{\circ} \mathrm{C}$ for $10 \mathrm{~min}$. The supernatants were collected, bathed at $37^{\circ} \mathrm{C}$ for $10 \mathrm{~min}$, then centrifuged $(13,000 \mathrm{rpm})$ at room temperature for $5 \mathrm{~min}$. The samples were divided into 2 layers. The bottom layer, containing cytoplasmic proteins, was collected and stored for further analysis. The bottom layer, containing membrane proteins, was dissolved in $500 \mu \mathrm{l}$ of ice-cold sterile water for $5 \mathrm{~min}$ at $4^{\circ} \mathrm{C}$, followed by a water bath at $37^{\circ} \mathrm{C}$ for $5 \mathrm{~min}$. After centrifugation $(13,000 \mathrm{rpm})$ at room temperature for $5 \mathrm{~min}$, the bottom layer was collected and dissolved in ice-cold sterile water again following the above steps. Finally, the membrane extracts were collected, and the protein concentration was measured using the BCA kit (Pierce, Rockford, IL, USA). For SDS-PAGE analysis, a total of $100 \mu 1$ membrane protein was mixed with $0.9 \mathrm{ml}$ acetone, incubated in an ice-cold condition for $20 \mathrm{~min}$ followed by centrifugation $(10,000 \mathrm{rpm})$ for $20 \mathrm{~min}$. The supernatants were removed, and $100 \mu \mathrm{l}$ of loading buffer and $2 \mu \mathrm{l}$ of $\beta$-mercaptoethanol were added to dissolve the sediments for sodium dodecyl sulfate polyacrylamide gel electrophoresis (SDS-PAGE) analysis. 


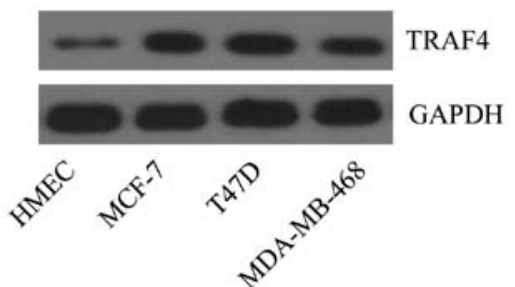

Figure 1. TRAF4 is overexpressed in human breast cancer cells. TRAF4 protein expression profiles in normal human mammary epithelial cells (HMECs) and human breast cancer cell lines MCF-7, T47D and MDA-MB-468 were examined by western blot analysis.

Co-immunoprecipitation. The cells were lysed and centrifuged, and extracts were then collected. Protein A-Sepharose beads (Amersham Biosciences AB, Uppsala, Sweden) mixed with a mouse monoclonal anti-Flag (Sigma, St. Louis, MO, USA), or mouse IgG as a control, were incubated at $4^{\circ} \mathrm{C}$ in $500 \mu \mathrm{l}$ of lysis buffer for $1 \mathrm{~h}$. Cell extracts were added to the prepared antibody-bead mixture and incubated at $4^{\circ} \mathrm{C}$ for $2 \mathrm{~h}$. Then, the bead complexes were collected and washed for a total of three times. Then, the protein complexes were eluted from the beads using glycine buffer ( $\mathrm{pH} 2.5$ ), and separated by SDS-PAGE and examined by western blotting.

Western blot analysis. Proteins were extracted from cells, and concentrations were measured using the BCA kit. A total of $20 \mu \mathrm{g}$ protein was separated by $12 \%$ SDS-PAGE electrophoresis followed by electro-blotting onto a nitrocellulose membrane (Amersham, Little Chalfont, UK). Then, the membrane was incubated in Tris-buffered saline (TBS) containing $2 \%$ non-fat dry milk to block non-specific binding at room temperature for $1 \mathrm{~h}$. The membrane was then washed with TBS and incubated with primary antibodies [antibodies against TRAF4, GAPDH, myc-tag, $\alpha$-tubulin and E-cadherin (all from Santa Cruz Biotechnology), phosphorylated Akt (Ser473), total Akt and Akt1 (all from Cell Signaling Technology, Danvers, MA, USA) diluted $(1: 1,000)]$ in the blocking buffer overnight at $4^{\circ} \mathrm{C}$. Subsequently, the membrane was washed with TBS. After that, the membrane was incubated in horseradish peroxidase (HRP)-conjugated secondary antibodies (Boster Corporation, Wuhan, China) diluted in blocking buffer for $1 \mathrm{~h}$. Finally, the membrane was washed followed by the addition of 4-chloro-1-naphthol (4-CN; $1 \mathrm{ml})$ with TBS $(9 \mathrm{ml})$ containing $6 \mu \mathrm{l}$ of $\mathrm{H}_{2} \mathrm{O}_{2}$, which was used for protein visualization.

Statistical analysis. Data are expressed as means \pm standard deviation (SD). The statistical significance of differences between two groups was determined by the Student's t-test, and among multiple groups was determined by one-way ANOVA. $\mathrm{P}<0.05$ was considered to indicate a statistically significant difference. All statistical analyses were performed using SPSS 11.5 Software (SPSS Inc., Chicago, IL, USA).

\section{Results}

TRAF4 is specifically overexpressed in human breast cancer cells. To verify the expression profiles of TRAF4 in human breast cancer, we delineated the protein expression level of
A

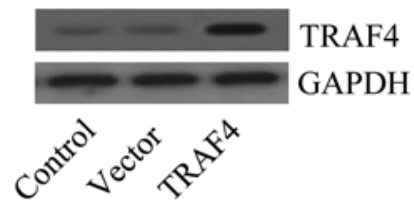

B

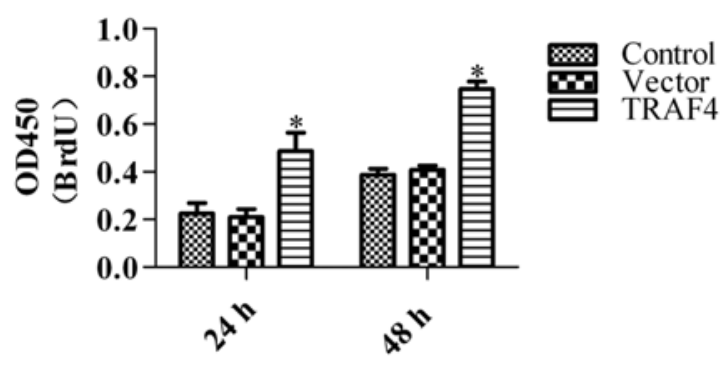

C

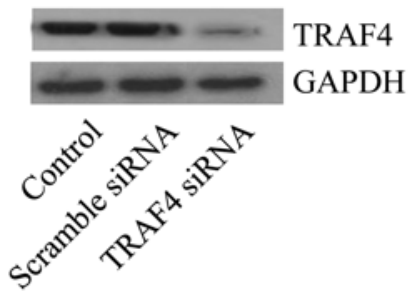

D

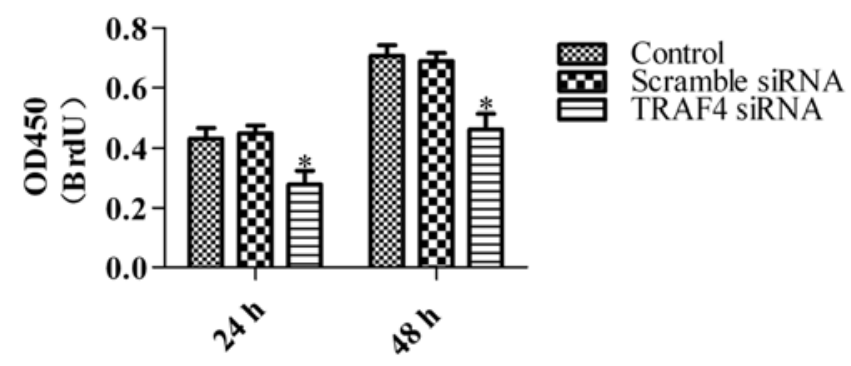

Figure 2. Effects of TRAF4 on cell proliferation. (A) Overexpression of TRAF4 in HMECs. Control, cells without treatment; vector, cells transfected with null vectors; TRAF4, cells transfected with TRAF4 overexpression vectors. (B) Proliferation of HMECs was analyzed by BrdU assay after 24 and $48 \mathrm{~h}$ of vectortransfection. (C) Knockdown of TRAF4 in MCF-7 breast cancer cells. Control, cells without treatment; Scramble siRNA, cells transfected with nonspecific scramble siRNA; TRAF4 siRNA, cells transfected with TRAF4 target siRNA. (D) Proliferation of MCF-7 cells was detected by BrdU assay 24 and $48 \mathrm{~h}$ after siRNA transfection. " $\mathrm{P}<0.05$ vs. negative control or positive control denotes a statistically significant difference.

TRAF4 in different breast cancer cell lines in vitro. The results showed that, compared with the normal human mammary epithelial cells (HMECs), TRAF4 was particularly overexpressed in the human breast cancer cell lines MCF-7, T47D and MDA-MB-468 (Fig. 1). The data indicate that TRAF4 has a critical role in human breast cancer development.

TRAF4 promotes cancer cell proliferation. To investigate the role of TRAF4 in breast cancer cells, we applied the TRAF4 overexpression vector or the TRAF target siRNA to 
A

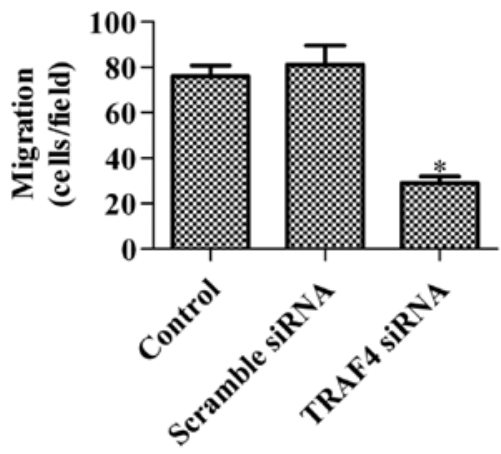

B

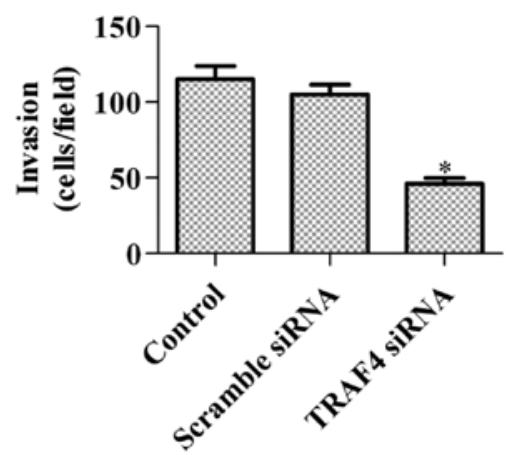

Figure 3. Effect of TRAF4 knockdown on tumor cell migration and invasion. Analysis of TRAF4 silencing on cell migration and invasion of MCF-7 cells Data are expressed as mean \pm standard deviation (SD), and differences were analyzed by one-way ANOVA. The means were obtained from three individual experiments. ${ }^{*} \mathrm{P}<0.05$.

A
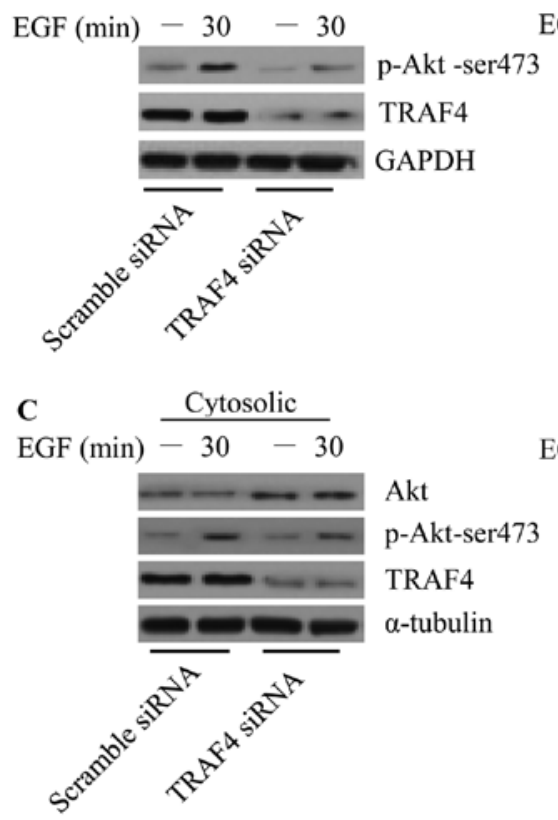

B

$\mathrm{EGF}(\min )-30-30$
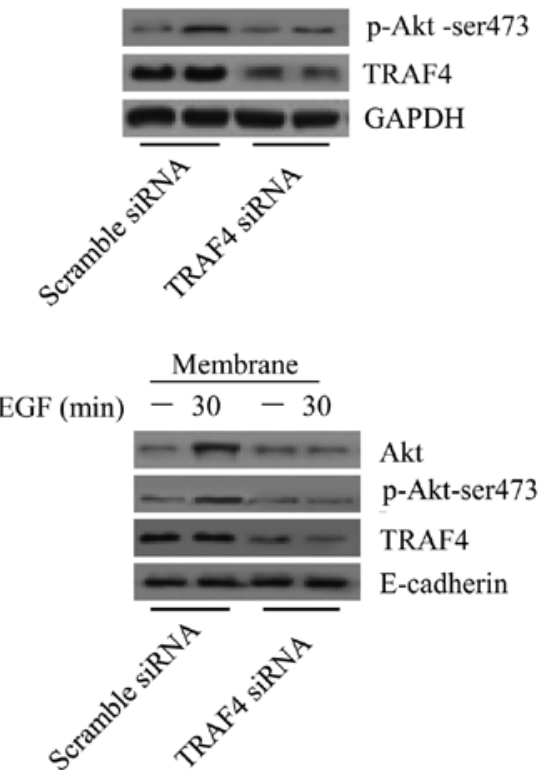

Figure 4. Effect of TRAF4 on Akt activation. Effect of TRAF4 knockdown on EGF-induced Akt phosphorylation in (A) MCF-7 and (B) T47 breast cancer cells. TRAF4 was silenced by siRNA and cells were starved for $24 \mathrm{~h}$. Then, EGF $(50 \mathrm{ng} / \mathrm{ml})$ was added and incubated for 30 min. Finally, cells were harvested for western blotting. (C) Examination of Akt membrane recruitment in siRNA-transfected MCF-7 cells. Cells were starved and treated with EGF (50 ng/ml) for $30 \mathrm{~min}$. Membrane and cytosolic fractions were isolated and analyzed by western blot analysis.

generate TRAF4-overexpressing or stable knockdown cell lines. We first generated TRAF4-overexpressing cells by transfecting HMECs with the TRAF4 overexpression vectors (Fig. 2A). The BrdU assay was used to determine the effects on cell proliferation. The results showed that overexpression of TRAF4 significantly promoted the proliferation of HMECs following transfection (Fig. 2B), whereas TRAF4 knockdown significantly inhibited cell proliferation in the breast cancer cell line MCF-7 (Fig. 2C and D). Similar results were obtained in the siRNA-transfected T47D and MDA-MB-468 cell lines (data not shown). These results imply that TRAF4 plays an important role in cancer cell proliferation.

Knockdown of TRAF4 impairs tumor cell migration and invasion. To further explore the function of TRAF4 in cancer cells, the role of TRAF on cell migration and invasion was determined. Knockdown of TRAF4 in MCF-7 cells significantly decreased the cell migratory and invasive abilities (Fig. 3A and $\mathrm{B})$. Furthermore, the same results were obtained when using the T47D and MDA-MB-468 cell lines (data not shown). Overall, the data suggest that TRAF4 has various tumorigenic roles.

TRAF4 increases Akt membrane recruitment and activation. To further investigate the underlying mechanism of TRAF4 in tumorigenesis, we aimed to identify the signaling pathway in which TRAF4 is involved. A previous study demonstrated that TRAF family members such as TRAF6 (27) are involved in the Akt pathway. As the TRAF family members share similar functional domains, we investigated whether TRAF4 
A

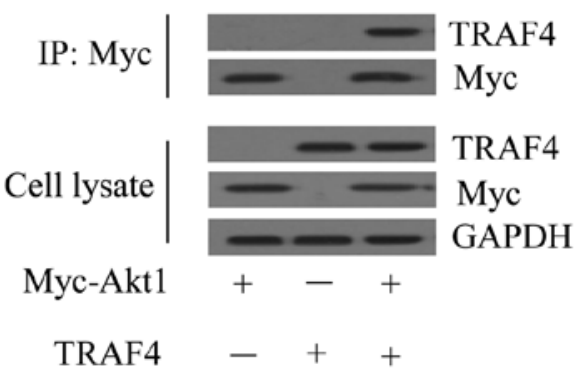

B

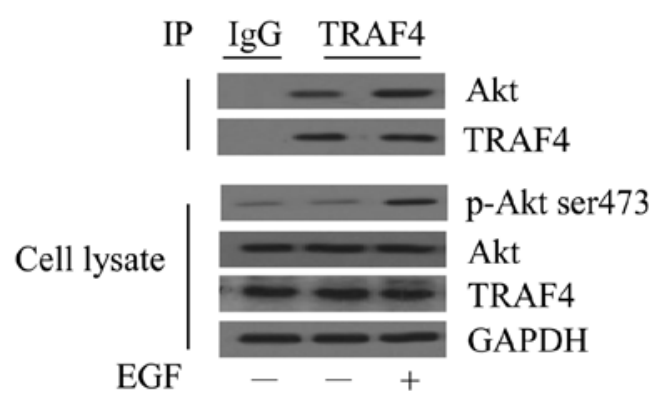

Figure 5. Detection of the interaction between TRAF4 and Akt. (A) Myc-Akt1 and TRAF4 expression vectors were co-transfected into $293 \mathrm{~T}$ cells. At $24 \mathrm{~h}$ after transfection, cell lysates were collected and immunoprecipitated with an anti-Myc antibody. (B) MCF-7 cells were starved and treated with EGF (50 ng/ $\mathrm{ml}$ ) for $30 \mathrm{~min}$. Cell extracts were immunoprecipitated with an anti-TRAF4 or IgG antibody. Western blot analysis was performed to detect target proteins with the indicated antibodies.

A

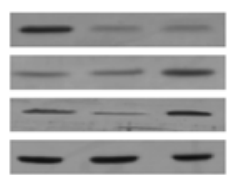

TRAF4

Akt

p-Akt ser473

GAPDH
B
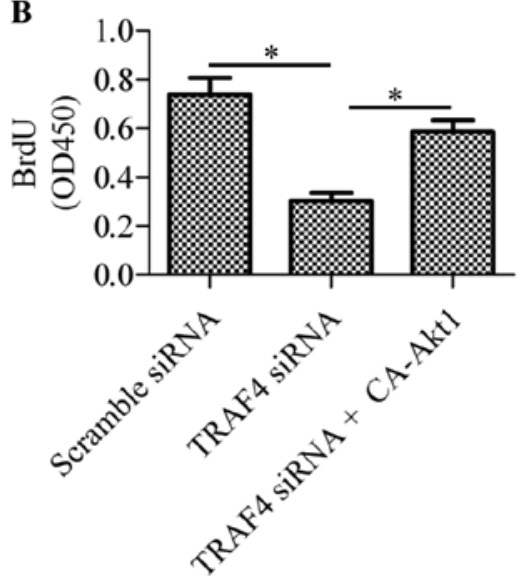

Figure 6. Effect of overexpression of constitutively active Akt on cell proliferation in TRAF4-knockdown cells. (A) CA-Akt1 plasmids were co-transfected with TRAF4 siRNA in MCF-7 cells. Cell extracts were collected at $48 \mathrm{~h}$, and western blotting was performed to detect the protein levels. (B) BrdU assay was used to determine cell proliferation in the CA-Akt1 and TRAF4 siRNA co-transfected cells. ${ }^{*} \mathrm{P}<0.05$ denotes a significant difference.

is capable of activating Akt. Upon EGF treatment, Akt phosphorylation (Ser473) was upregulated in the control cells. However, EGF-induced phosphorylation of Akt was markedly inhibited in the TRAF4-knockdown MCF-7 (Fig. 4A) and T47D breast cancer cells (Fig. 4B). Akt membrane recruitment is critical for Akt activation. We next examined whether TRAF4 plays an important role in Akt membrane translocation. The results showed that knockdown of TRAF4 caused Akt cytosolic accumulation, and the phosphorylation of Akt was markedly disturbed both in the cytoplasm and membrane (Fig. 4C). The data imply that TRAF4 plays an important role in Akt membrane recruitment in breast cancer cells.

TRAF4 displays an interaction with Akt. To explore the underlying mechanism of TRAF4 in regulating Akt, we examined whether a direct interaction is observed between the two proteins. Myc-Akt1 and TRAF4 were co-transfected in 293T cells and immunoprecipitated with the anti-Myc antibody. The results showed that the TRAF4 protein was only detected in the immunoprecipitation complex from the Myc-Akt1 and TRAF4 co-transfected cells (Fig. 5A). Next, we aimed to determine the interaction of endogenous Akt1 and TRAF4 in the MCF-7 cells. The results showed that endogenous Akt interacted with TRAF4 in breast cancer cells and the interaction was enhanced upon EGF treatment (Fig. 5B). These data indicate that TRAF4 undergoes an interaction with Akt in breast cancer cells.

TRAF4 promotes cell proliferation through Akt. To further confirm the function of TRAF4 in the Akt signaling pathway, we transfected constitutively activated Akt1 (CA-Akt1) into TRAF4-knockdown MCF-7 breast cancer cells. The results showed that transfection of CA-Akt1 significantly upregulated the phosphorylation of Akt in the TRAF4-knockdown cells (Fig. 6A). Cell growth arrest induced by TRAF4 silencing was reversed by CA-Akt1 transfection (Fig. 6B). These data suggest that TRAF4 regulation on cell proliferation is dependent on Akt activity.

\section{Discussion}

Breast cancer is currently the second leading cause of cancer-related death, and is the most commonly diagnosed cancer in women worldwide (28). Although a better understanding of the biological mechanisms that underlie breast cancer development has been achieved with the development 
of advanced molecular biology techniques in recent years, a major current challenge in the treatment of breast cancer is to discover novel and effective targets that can complement current therapies. In the present study, we demonstrated that TRAF4 plays an important role in the activation of the Akt signaling pathway in breast cancer. Considering the important function of TRAF4 in the development of breast cancer, TRAF4 may be regarded as a potential target for breast cancer therapy.

Our data revealed that TRAF4 was overexpressed in breast cancer cell lines. Moreover, RNAi-mediated knockdown of TRAF4 significantly inhibited cell proliferation, invasive and migratory abilities of breast cancer cells. These results suggest that TRAF4 plays an important role in human breast cancers. Although TRAF4 overexpression in a wide range of human cancers has been verified (14), TRAF4 overexpression in squamous cell carcinoma of the head and neck has an antitumor effect through the induction of cell apoptosis and the suppression of colony formation $(29,30)$. It has been demonstrated that TRAF4 is a p53-regulated pro-apoptotic gene in p53 temperature-sensitive cells (31). In contrast, a recent study demonstrated that TRAF4 was a downstream gene of steroid receptor coactivator 3 (SRC-3), which is an oncogenic nuclear receptor coactivator that increases p53 destabilization leading to resistance to cytotoxic stress and poor prognosis in breast cancer patients (20). Therefore, it seems that TRAF4 overexpression has different outcomes in different cancers. The apparent discrepancy implies that TRAF4 may have different biological functions upon stimulation and cell type.

More recently, TRAF4 has been suggested to participate in breast cancer migration through destabilizing tight junctions in mammary epithelial cells (32). Wang et al showed that Smad ubiquitin regulatory factors (Smurfs) promoted the ubiquitination of TRAF4 which is essential for the proper localization of TRAF4 to tight junctions in confluent epithelial cells (33). In prostate cancer, TRAF4 expression was found to be regulated by tumor-suppressor microRNA-29a, and an inverse correlation was identified in tumor tissues from radical prostatectomy (34). In addition, Zhang et al demonstrated that TRAF4 promotes the transforming growth factor (TGF)- $\beta$ signaling pathway, which contributes to the pathogenesis of breast cancer (35). All of these data indicate that TRAF4 plays a critical role in tumorigenesis. In the present study, we found that TRAF4 regulated the activation of Akt in breast cancer cells. Our data are consistent with a more recent study which indicated that TRAF4, possessing ubiquitin-protein ligase activity, activates Akt through the ubiquitination of Akt in lung cancer (36).

It is well-known that the Akt pathway is closely related to tumorigenesis by regulating cell growth and survival, cell cycle and metabolism (37-39). Akt is deregulated in many types of cancer, including breast cancer, and contributes to cancer cell growth and survival, and resistance to chemotherapy or radiotherapy $(40,41)$. Akt activation is dependent on the phosphorylation of Thr308 and Ser473 upon various stimuli such as insulin and epidermal growth factor (EGF) (42). Studies suggest that Akt recruitment to the membrane upon growth factor stimuli is essential for activation $(27,43)$. In the present study, we found that TRAF4 regulated Akt membrane recruitment in breast cancer cells upon EGF stimulation, whereas knockdown of TRAF4 caused a decline in Akt membrane recruitment and activation. We further revealed a direct interaction between TRAF4 and Akt, which was the basis for TRAF4 in promoting Akt membrane recruitment. In accordance with this, Li et al demonstrated that TRAF4 functions as an E3 ligase which regulates Akt ubiquitination and activation (36). Previous studies suggest that Lys63-mediated ubiquitination of Akt is necessary for Akt activation (44). However, we did not further investigate the ubiquitination of Akt by TRAF4 activity in breast cancer cells. Whether TRAF4 promotes Akt activation by ubiquitination in breast cancer cells therefore remains uncertain. Additionally, we found that overexpression of constitutively active Akt1 reversed the cell growth arrest caused by TRAF4 knockdown, implying that TRAF4 regulates cell growth through the Akt signaling pathway.

Taken together, we found that TRAF4 promoted Akt activation in human breast cancer cells. Given the important roles of Akt in regulating tumorigenesis, targeting TRAF4 to inhibit activated Akt in breast cancer is a potential therapeutic strategy for the prevention and treatment of human breast cancers.

\section{Acknowledgements}

This research was supported in part by the Natural Science Foundation (no. 2011A310005), the Science and Technique Foundation (no. 112102310206) and a University Key Teacher grant from the Ministry of Education (no. 2012GGJS-136) of Henan.

\section{References}

1. Inoue $\mathrm{J}$, Ishida $\mathrm{T}$, Tsukamoto $\mathrm{N}$, et al: Tumor necrosis factor receptor-associated factor (TRAF) family: adapter proteins that mediate cytokine signaling. Exp Cell Res 254: 14-24, 2000.

2. Arron JR, Walsh MC and Choi Y: TRAF-mediated TNFR-family signaling. Curr Protoc Immunol: Nov 1, 2002, Chapter 11: Unit 11.9D. doi: 10.1002/0471142735.im1109ds51.

3. Xie P: TRAF molecules in cell signaling and in human diseases. J Mol Signal 8: 7, 2013.

4. Keats JJ, Fonseca R, Chesi M, et al: Promiscuous mutations activate the noncanonical NF-kappaB pathway in multiple myeloma. Cancer Cell 12: 131-144, 2007.

5. Namjou B, Choi CB, Harley IT, et al: Evaluation of TRAF6 in a large multiancestral lupus cohort. Arthritis Rheum 64: 1960-1969, 2012

6. Zucchelli S, Codrich M, Marcuzzi F, et al: TRAF6 promotes atypical ubiquitination of mutant DJ-1 and alpha-synuclein and is localized to Lewy bodies in sporadic Parkinson's disease brains. Hum Mol Genet 19: 3759-3770, 2010.

7. Kedinger $\mathrm{V}$ and Rio MC: TRAF4, the unique family member. Adv Exp Med Biol 597: 60-71, 2007.

8. Masson R, Regnier CH, Chenard MP, et al: Tumor necrosis factor receptor associated factor 4 (TRAF4) expression pattern during mouse development. Mech Dev 71: 187-191, 1998.

9. Kedinger V, Alpy F, Tomasetto C, et al: Spatial and temporal distribution of the traf4 genes during zebrafish development. Gene Expr Patterns 5: 545-552, 2005.

10. Blaise S, Kneib M, Rousseau A, et al: In vivo evidence that TRAF4 is required for central nervous system myelin homeostasis. PLoS One 7: e30917, 2012.

11. Regnier CH, Masson R, Kedinger V, et al: Impaired neural tube closure, axial skeleton malformations, and tracheal ring disruption in TRAF4-deficient mice. Proc Natl Acad Sci USA 99: 5585-5590, 2002.

12. Shiels H, Li X, Schumacker PT, et al: TRAF4 deficiency leads to tracheal malformation with resulting alterations in air flow to the lungs. Am J Pathol 157: 679-688, 2000. 
13. Cherfils-Vicini J, Vingert B, Varin A, et al: Characterization of immune functions in TRAF4-deficient mice. Immunology 124: $562-574,2008$.

14. Camilleri-Broët S, Cremer I, Marmey B, et al: TRAF4 overexpression is a common characteristic of human carcinomas. Oncogene 26: 142-147, 2007.

15. Rhodes DR, Yu J, Shanker K, et al: Large-scale meta-analysis of cancer microarray data identifies common transcriptional profiles of neoplastic transformation and progression. Proc Natl Acad Sci USA 101: 9309-9314, 2004.

16. Regnier CH, Tomasetto $\mathrm{C}$, Moog-Lutz C, et al: Presence of a new conserved domain in CART1, a novel member of the tumor necrosis factor receptor-associated protein family, which is expressed in breast carcinoma. J Biol Chem 270: 25715-25721, 1995.

17. Bieche I, Tomasetto $\mathrm{C}$, Regnier $\mathrm{CH}$, et al: Two distinct amplified regions at 17q11-q21 involved in human primary breast cancer. Cancer Res 56: 3886-3890, 1996.

18. Glauner H, Siegmund D, Motejadded H, et al: Intracellular localization and transcriptional regulation of tumor necrosis factor (TNF) receptor-associated factor 4 (TRAF4). Eur J Biochem 269: 4819-4829, 2002.

19. Kedinger V, Alpy F, Baguet A, et al: Tumor necrosis factor receptor-associated factor 4 is a dynamic tight junction-related shuttle protein involved in epithelium homeostasis. PLoS One 3: e3518, 2008.

20. Yi P, Xia W, Wu RC, et al: SRC-3 coactivator regulates cell resistance to cytotoxic stress via TRAF4-mediated p53 destabilization. Genes Dev 27: 274-287, 2013.

21. Krajewska M, Krajewski S, Zapata JM, et al: TRAF-4 expression in epithelial progenitor cells. Analysis in normal adult, fetal, and tumor tissues. Am J Pathol 152: 1549-1561, 1998.

22. Ye X, Mehlen P, Rabizadeh S, et al: TRAF family proteins interact with the common neurotrophin receptor and modulate apoptosis induction. J Biol Chem 274: 30202-30208, 1999.

23. Takeshita F, Ishii KJ, Kobiyama K, et al: TRAF4 acts as a silencer in TLR-mediated signaling through the association with TRAF6 and TRIF. Eur J Immunol 35: 2477-2485, 2005.

24. Esparza EM and Arch RH: TRAF4 functions as an intermediate of GITR-induced NF-kappaB activation. Cell Mol Life Sci 61: 3087-3092, 2004.

25. Abell AN and Johnson GL: MEKK4 is an effector of the embryonic TRAF4 for JNK activation. J Biol Chem 280: 35793-35796, 2005.

26. Zepp JA, Liu C, Qian W, et al: Cutting edge: TNF receptor-associated factor 4 restricts IL-17-mediated pathology and signaling processes. J Immunol 189: 33-37, 2012.

27. Yang WL, Wang J, Chan $\mathrm{CH}$, et al: The E3 ligase TRAF6 regulates Akt ubiquitination and activation. Science 325: 1134-1138, 2009.
28. Siegel R, Naishadham D and Jemal A: Cancer statistics for Hispanics/Latinos, 2012. CA Cancer J Clin 62: 283-298, 2012.

29. Gu X, Coates PJ, MacCallum SF, et al: TRAF4 is potently induced by TAp63 isoforms and localised according to differentiation in SCCHN. Cancer Biol Ther 6: 1986-1990, 2007.

30. Rozan LM and El-Deiry WS: Identification and characterization of proteins interacting with Traf4, an enigmatic p53 target. Cancer Biol Ther 5: 1228-1235, 2006.

31. Sax JK and El-Deiry WS: Identification and characterization of the cytoplasmic protein TRAF4 as a $\mathrm{p} 53$-regulated proapoptotic gene. J Biol Chem 278: 36435-36444, 2003.

32. Rousseau A, McEwen AG, Poussin-Courmontagne P, et al: TRAF4 is a novel phosphoinositide-binding protein modulating tight junctions and favoring cell migration. PLoS Biol 11: e1001726, 2013.

33. Wang X, Jin C, Tang Y, Tang LY and Zhang YE: Ubiquitination of tumor necrosis factor receptor-associated factor 4 (TRAF4) by Smad ubiquitination regulatory factor 1 (Smurf1) regulates motility of breast epithelial and cancer cells. J Biol Chem 288: 21784-21792, 2013.

34. Ahmed F, Shiraishi T, Vessella RL and Kulkarni P: Tumor necrosis factor receptor associated factor-4: an adapter protein overexpressed in metastatic prostate cancer is regulated by microRNA-29a. Oncol Rep 30: 2963-2968, 2013.

35. Zhang L, Zhou F, Garcia de Vinuesa A, et al: TRAF4 promotes TGF- $\beta$ receptor signaling and drives breast cancer metastasis. Mol Cell 51: 559-572, 2013

36. Li W, Peng C, Lee MH, et al: TRAF4 Is a critical molecule for Akt activation in lung cancer. Cancer Res 73: 6938-6950, 2013.

37. Brazil DP, Park $J$ and Hemmings BA: PKB binding proteins. Getting in on the Akt. Cell 111: 293-303, 2002.

38. Manning BD and Cantley LC: AKT/PKB signaling: navigating downstream. Cell 129: 1261-1274, 2007.

39. Liu P, Cheng H, Roberts TM and Zhao JJ: Targeting the phosphoinositide 3-kinase pathway in cancer. Nat Rev Drug Discov 8: 627-644, 2009.

40. Bose S, Chandran S, Mirocha JM and Bose N: The Akt pathway in human breast cancer: a tissue-array-based analysis. Mod Pathol 19: 238-245, 2006.

41. Stal O, Perez-Tenorio G, Akerberg L, et al: Akt kinases in breast cancer and the results of adjuvant therapy. Breast Cancer Res 5: R37-R44, 2003.

42. Hay N: The Akt-mTOR tango and its relevance to cancer. Cancer Cell 8: 179-183, 2005.

43. Brazil DP and Hemmings BA: Ten years of protein kinase B signalling: a hard Akt to follow. Trends Biochem Sci 26: 657-664, 2001.

44. Yang WL, Wu CY, Wu J and Lin HK: Regulation of Akt signaling activation by ubiquitination. Cell Cycle 9: 487-497, 2010. 